\title{
CHANNELING OF CHARGED PARTICLES THROUGH PERIODICALLY BENT CRYSTALS: ON THE POSSIBILITY OF A GAMMA LASER *
}

\author{
A. V. KOROL, W. KRAUSE, A. V. SOLOV'YOV AND W. GREINER \\ Institut für Theoretische Physik der Johann Wolfgang Goethe-Universität, 60054 \\ Frankfurt am Main, Germany \\ E-mail: solovyov@th.physik.uni-frankfurt.de
}

\begin{abstract}
We discuss radiation generated by positrons channeling in a crystalline undulator. The undulator is produced by periodically bending a single crystal with an amplitude much larger than the interplanar spacing. Different approaches for bending the crystal are described and the restrictions on the parameters of the bending are established. We present the results of numeric calculations of the spectral distributions of the spontaneous emitted radiation and estimate the conditions for stimulated emission. Our investigations show that the proposed mechanism provides an efficient source for high energy photons, which is worth to be studied experimentally.
\end{abstract}

\section{Introduction}

We discuss a new mechanism, initially proposed in 1 , f for the generation of high energy photons by means of the planar channeling of ultra-relativistic positrons through a periodically bent crystal. In this system there appears, in addition to the well-known channeling radiation, an undulator type radiation due to the periodic motion of the channeling positrons which follow the bending of the crystallographic planes. The intensity and the characteristic frequencies of this undulator radiation can be easily varied by changing the positrons energy and the parameters of the crystal bending.

The mechanism of the photon emission by means of the crystalline undulator is illustrated in Figure 1. It is important to stress that we consider the case when the amplitude $a$ of the bending is much larger than the interplanar spacing $d\left(\sim 10^{-8} \mathrm{~cm}\right)$ of the crystal $(a \sim 10 d)$, and, simultaneously, is much less than the period $\lambda$ of the bending $\left(a \sim 10^{-5} \ldots 10^{-4} \lambda\right)$.

In addition to the spontaneous photon emission by the crystalline undulator, the scheme we propose leads to a possibility to generate stimulated emission. This is due to the fact, that photons emitted at the points of the maximum curvature of the trajectory travel almost parallel to the beam and

${ }^{*}$ CONTRIBUTED TO THE CONFERENCE "FUNDAMENTAL AND APPLIED ASPECTS OF MODERN PHYSICS" IN LDERITZ, NAMIBIA, 2001 


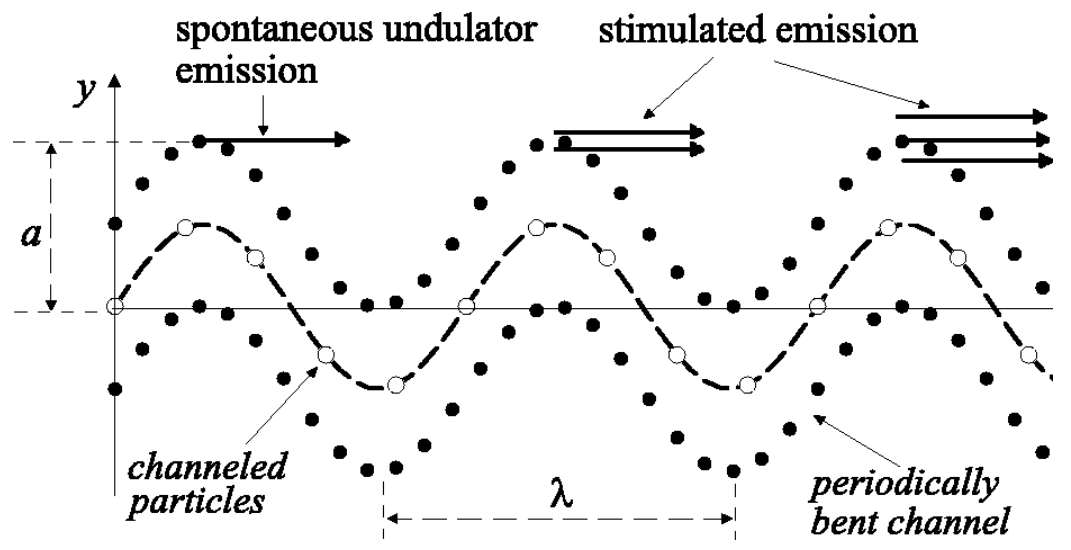

Figure 1. Schematic representation of spontaneous and stimulated radiation due to positrons channeling in a periodically bent crystal. The $y$ - and $z$-scales are incompatible!

thus, stimulate the photon generation in the vicinity of all successive maxima and minima of the trajectory.

\section{The bent crystal}

The bending of the crystal can be achieved either dynamically or statically. In 4. it was proposed to use a transverse acoustic wave to dynamically bend the crystal. The important feature of this scheme is that the time period of the acoustic wave is much larger than the time of flight $\tau$ of a bunch of positrons through the crystal. Then, on the time scale of $\tau$, the shape of the crystal bending doesn't change, so that all particles of the bunch channel inside the same undulator. One possibility to couple the acoustic waves to the crystal is to place a piezo sample atop the crystal and to use radio frequency to excite oscillations.

The tially in 4 a and later in $\mathrm{O}_{\text {. In }}$ Ine latter work the idea to construct a crystalline undulator based on graded composition strained layers was suggested.

Let us now consider the conditions to be fulfiled in the stable channeling regime. The channeling process in a periodically bent crystal takes place if the maximum centrifugal force in the channel, $F_{\mathrm{cf}} \approx m \gamma c^{2} / R_{\min }\left(R_{\min }\right.$ being the minimum curvature radius of the bent channel), is less than the maximal force due to the interplanar field, $F_{\text {int }}$ which is equal to the maximum gradient of 


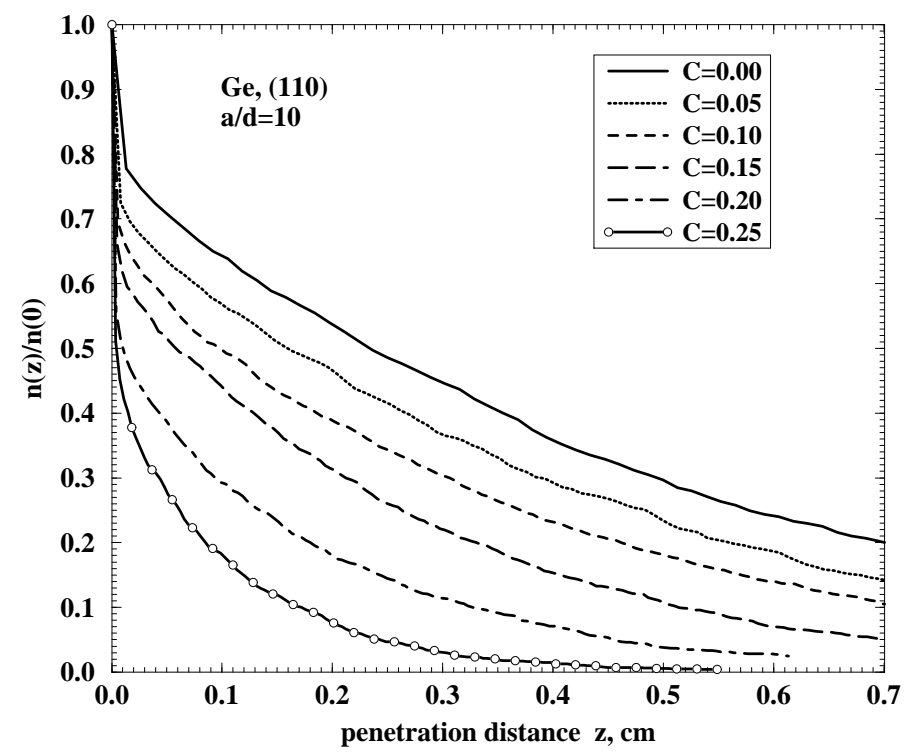

Figure 2. The calculated beam dencity dependences $n(z) / n(0)$ versus penetration distance $z$ for $5 \mathrm{GgV}$ positrons channeling along the (110) in Ge crystal for various values of the parameter $C$ 自. The data correspond to the shape function of the channel: $S(z)=a \sin (2 \pi z / \lambda)$. The $a / d$ ratio equals 10 . For each indicated $C$ the corresponding values of $\lambda$, and the calculated magnitudes of the dechanneling lengths $L_{d}^{c}$ and the number of undulator periods $N_{d}^{c}=L_{d}^{c} / \lambda$ are presented in Table 1 .

the interplanar field (see 2). More specifically, the ratio $C=F_{\text {cf }} / F_{\text {int }}$ is better to keep smaller than 0.1 , because otherwise the phase volume of channeling trajectories becomes significantly reduced (see also 4 ). The inequality $C<$ 0.1 connects the energy of the particle, $\varepsilon=m \gamma c^{2}$, the parameters of the bending (these enter through the quantity $R_{\min }$ ), and the characteristics of the crystallographic plane.

A particle channeling in a crystal (straight or bent) undergoes scattering by electrons and nuclei of the crystal. These random collisions lead to a gradual increase of the particle energy associated with the transverse oscillations in the channel. As a result, the transverse energy at some distance $L_{d}$ from the entrance point exceeds the depth of the interplanar potential well, and 


$\begin{array}{rrrrrrrrr}\text { Crystal: } & \text { Ge, } & d= & 2.00 \AA, & R_{c}= & 0.42 \mathrm{~cm} & & & \\ C & R_{\text {min }} & \lambda & L_{d}^{e} & L_{d}^{c} & N_{d}^{e} & N_{d}^{c} & \omega_{1} & p \\ & \mathrm{~cm} & \mu \mathrm{m} & \mathrm{cm} & \mathrm{cm} & & & \mathrm{MeV} & \\ 0.00 & \infty & - & 0.263 & 0.513 & - & - & - & - \\ 0.05 & 8.465 & 81.8 & 0.237 & 0.450 & 29 & 55 & 1.37 & 1.50 \\ 0.10 & 4.232 & 57.8 & 0.213 & 0.364 & 36 & 63 & 1.26 & 2.13 \\ 0.15 & 2.822 & 47.2 & 0.190 & 0.269 & 40 & 57 & 1.15 & 2.61 \\ 0.20 & 2.116 & 40.9 & 0.168 & 0.176 & 41 & 43 & 1.05 & 3.01 \\ 0.25 & 1.693 & 36.6 & 0.148 & 0.095 & 40 & 26 & 0.98 & 3.36 \\ 0.30 & 1.411 & 33.4 & 0.129 & 0.060 & 38 & 18 & 0.92 & 3.68 \\ 0.35 & 1.209 & 30.9 & 0.111 & 0.028 & 35 & 9 & 0.86 & 3.98 \\ 0.40 & 1.058 & 28.9 & 0.095 & 0.012 & 32 & 4 & 0.82 & 4.25\end{array}$

Table 1. Dechanneling lengths for $5 \mathrm{GeV}$ positron channeling along the (110) planes for the Ge crystal and various values of the parameter $C$ 8. The data correspond to the shape function $S(z)=a \sin (2 \pi z / \lambda)$. The $a / d$ ratio equals 10 except for the case $C=0$ (the straight channel). The quantity $L_{d}^{c}$ presents the accurately calculated dechanneling length 8, $N_{d}^{c}=L_{d}^{c} / \lambda$ is the corresponding number of the undulator periods, $L_{d}^{e}$ is the dechanneling length derived from a simple estimate (see 6.78), $N_{d}^{e}=L_{d}^{e} / \lambda$. Other parameters are: $d$ is the interplanar spacing, $R_{c}=\varepsilon / U_{\max }^{\prime}$ is the critical (minimal) radius consistent with the condition $C \ll 1, \omega_{1}$ is the energy of the first harmonic of the crystalline undulator radiation for the forward emission, $p$ is the undulator parameter. $R_{\min }$ is the minimum curvature radius of the bent channel centerline.

the pafticle leaves the channel. The quantity $L_{d}$ is called the dechanneling length 5 . To estimate $L_{d}$ one may follow the method described in 6.6.8. Thus, to consider the undulator radiation formed in a crystalline undulator, it is meaningful to assume that the crystal length does not exceed $L_{d}$. The detailed numerical analysis of the dechanneling phenomena in periodically bent crystals and its influence on the spectral characteristics of the undulator radiation has been performed in $\mathrm{B}$. An example of these calculations for $5 \mathrm{GeV}$ positrons channeling along the (110) in Ge crystal is presented in figure 2 and in 11. In $\mathrm{B}$, the similar calculations have been also performed for the $S i$ and $W$ crystals.

Let us demonstrate how one can estimate, for a given crystal and energy $\varepsilon$, the ranges of the parameters $a$ and $\lambda$ which are subject to the conditions formulated above. For doing this we assume that the shape of the centerline of the periodically bent crystal is $a \sin (2 \pi z / \lambda)$. Figure 3 illustrates the above mentioned restrictions in the case of $\varepsilon=0.5 \mathrm{GeV}$ positrons planar channeling in $\mathrm{Si}$ along the (110) crystallographic planes. The diagonal straight lines correspond to various values (as indicated) of the parameter $C$. The curved 


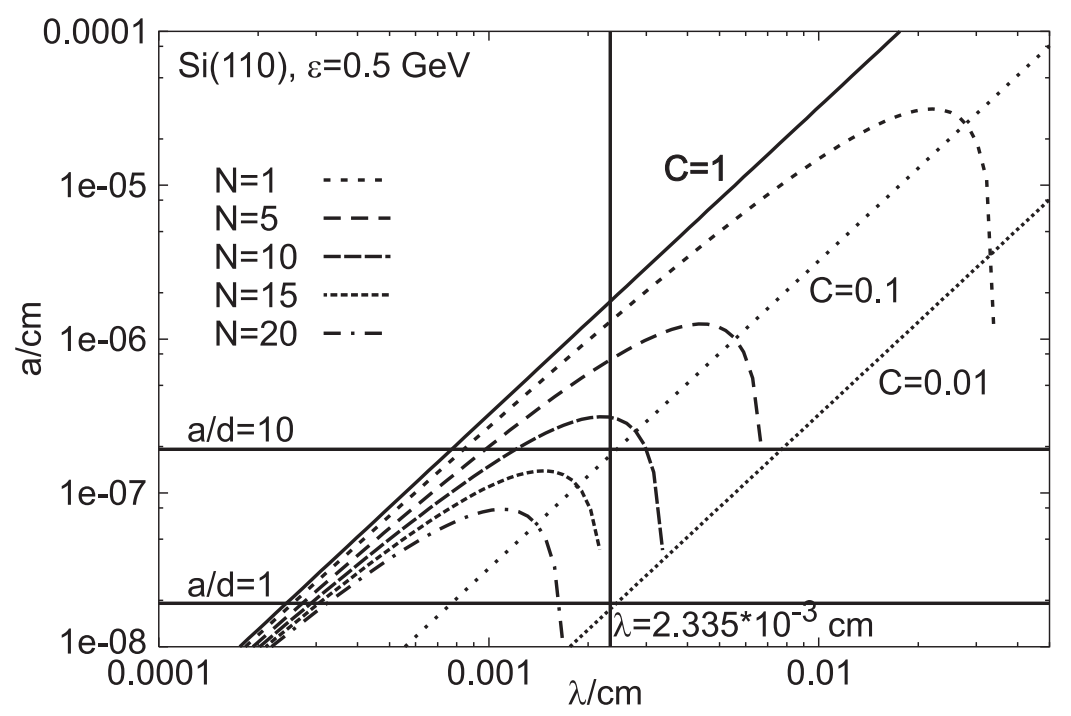

Figure 3. The range of parameters $a$ and $\lambda$ for a bent $\operatorname{Si}(110)$ crystal at $\varepsilon=500 \mathrm{MeV}$.

lines correspond to various values (as indicated) of the number of undulator periods $N$ related to the dechanneling length $L_{d}$ through $N=L_{d} / \lambda$. The horizontal lines mark the values of the amplitude equal to $d$ (with $d=1.92$. $10^{-8} \mathrm{~cm}$ being the (110) interplanar distance in $\mathrm{Si}$ ) and to $10 \mathrm{~d}$. The vertical line marks the value $\lambda=2.335 \cdot 10^{-3} \mathrm{~cm}$, for which the spectra (see section 3) were calculated.

\section{Spectra of the spontaneous emitted radiation}

Let us consider the spectra of the spontaneous emitted radiation calculated in $\mathrm{Z}$ using the quasiclassical method $\mathrm{E}$. In the trajectories of the particles were calculated numerically and then the spectra were evaluated. The latter include both radiation mechanisms, the undulator and the channeling radiation.

The spectral distributions of the total radiation emitted in forward direction for $\varepsilon=500 \mathrm{MeV}$ positrons channeling in Si along the (110) crystallographic planes are plotted in figure 4 . The wavelength is fixed at $\lambda=2.335 \cdot 10^{-3} \mathrm{~cm}$, while the ratio $a / d$ is changed from 0 to 10 . The length of the crystal is $L_{d}=3.5 \cdot 10^{-2} \mathrm{~cm}$ and corresponds to $N=15$ undulator periods. The first graph in figure 1 corresponds to the case of the straight 

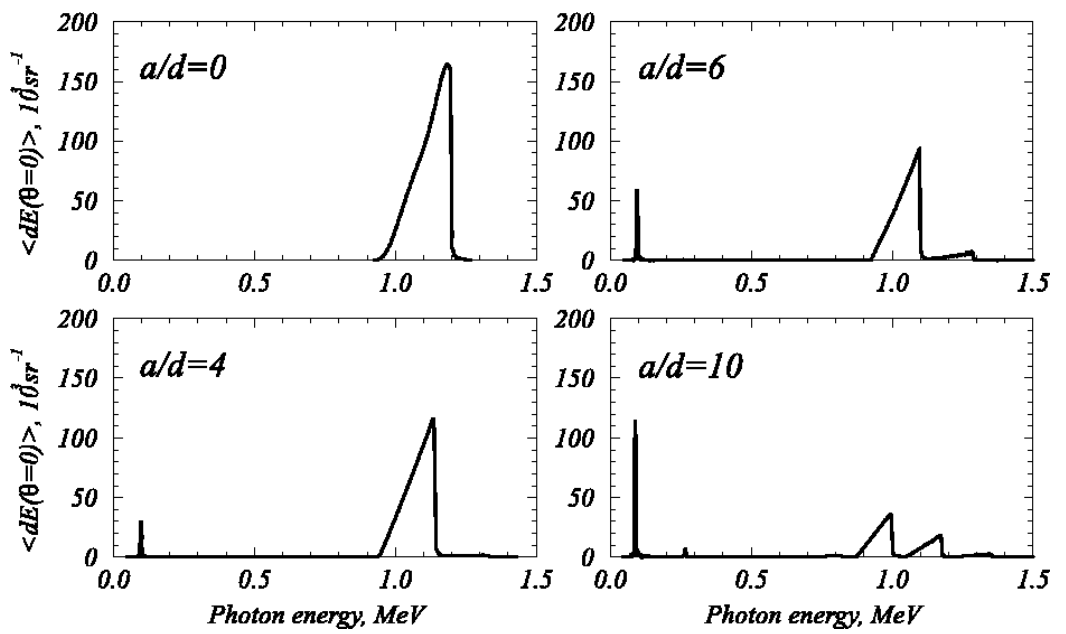

Figure 4. Spectral distributions of the total radiation emitted in forward direction for $\varepsilon=$ $500 \mathrm{MeV}$ positrons channeling in Si along the (110) crystallographic planes for different $a / d$ ratios.

channel $(a / d=0)$ and, hence, presents the spectral dependence of the ordinary channeling radiation only. The spectrum starts at $\hbar \omega \approx 960 \mathrm{keV}$, reaches its maximum value at $1190 \mathrm{keV}$, and steeply cuts off at $1200 \mathrm{keV}$. This peak corresponds to the radiation into the first harmonic of the ordinary channeling radiation, and there is almost no radiation into higher harmonics. The latter fact is consistent with general theory of dipole radiation by ultra-relativistic particles undergoing quasiperiodic motion. The dipole approximation is valid provided the corresponding undulator parameter $p_{c}=2 \pi \gamma\left(a_{c} / \lambda_{c}\right)$ is much less than 1 . In this relation $a_{c}$ and $\lambda_{c}$ stand for the characteristic scales of, correspondingly, the amplitude and the wavelength of the quasiperiodic trajectory. In the case of $0.5 \mathrm{GeV}$ positrons channeled along the (110) planes in Si one has $p_{c} \approx 0.2 \ll 1$ and all the channeling radiation is concentrated within some interval in the vicinity of the energy of the first harmonic.

Increasing the $a / d$ ratio leads to modifications in the spectrum of radiation. The changes which occur manifest themselves via three main features, (i) the lowering of the ordinary channeling radiation peak, (ii) the gradual increase of the intensity of undulator radiation due to the crystal bending, (iii) the appearing of additional structure (the sub-peaks) in the vicinity of the first harmonic of the ordinary channeling radiation. A more detailed analysis of these spectra can be found in $\mathrm{Q}$. 


\section{Discussion of stimulated photon emission}

The scheme illustrated by figure 1 allows to discuss the possibility to generate stimulated emission of high energy photons by means of a bunch of ultra-relativistic positrons moving in a periodically bent channel. Indeed the photons emitted in the nearly forward direction at some maximum or minimum point of the trajectory by a group of particles of the bunch stimulate the emission of photons with the same energy by another (succeeding) group of particles of the same bunch when it reaches the next maximum/minimum.

In 3 estimates for the gain factor for the spontaneous emission in crystalline undulators were obtained. It was demonstrated that to achieve a total gain equal to 1 on the scale of the crystal length (equal to the dechanneling length), one has to consider volume densities $n$ of the channeling positrons on the level of $10^{20} \ldots 10^{21} \mathrm{~cm}^{-3}$ for positron energies within the range $0.5 \ldots 5$ $\mathrm{GeV}$. These magnitudes are high enough to be questioned whether they can be really reached.

Let us estimate the volume density $n$ of a positron bunch which can be achieved in modern colliders. To do this we use the data presented in 10 (see p.142) for a beam of $50 \mathrm{GeV}$ positrons available at SLC (SLAC, 1989). The bunch length is $0.1 \mathrm{~cm}$ and the beam radius is $1.5 \mu \mathrm{m}(\mathrm{H})$ and 0.5 $\mu \mathrm{m}(\mathrm{V})$, resulting in the volume of one bunch $V=2.4 \cdot 10^{-9} \mathrm{~cm}^{3}$. The number of particles per bunch is given as $4.0 \cdot 10^{10}$. Therefore, one obtains $n=1.7 \cdot 10^{19} \mathrm{~cm}^{-3}$. This value, although being lower by an order of magnitude than the estimates obtained in $\mathrm{E}$, shows that the necessary densities should be reachable in the future with accelerators optimized for high particle densities.

Finally let us discuss the required transverse emittance of the beam. For doing this we need to consider the angle between the particle's trajectory and the crystal plane. If this angle is larger than the Lindhard angle $\Psi_{\mathrm{P}}$, the particle will not be captured into the channeling mode and leaves the channel immediately 11 . For $5 \mathrm{GeV}$ positrons channeling along the (110) plane of silicon, we have $\Psi_{\mathrm{P}}=72 \mu \mathrm{rad}$ and for $50 \mathrm{GeV}$ positrons it equals to $\Psi_{\mathrm{P}}=23 \mu \mathrm{rad}$.

One can compare these values with the divergence of the SLC beam, which transverse emittance in vertical direction is given as $0.05 \pi \mathrm{rad} \mathrm{nm} 0$. With a vertical beam radius of $0.5 \mu \mathrm{m}$ we get for the vertical beam divergence $\Psi=100 \mu \mathrm{rad}$. Thus the divergence of the beam is about four times higher than the acceptance of the channel and so only a quarter of all particles will participate in the channeling process. Evidently it is necessary to reduce the divergence of the beam, for example by increasing the beam radius. But then it is also necessary to reduce the bunch length, to keep the particle density

luederitz: submitted to World Scientific on November 13, 2018 
high enough. Fortunately, like for the particle density, the values achievable today differ only about one order of magnitude from the values estimated above for the stimulated emission.

\section{Conclusion}

To conclude we point out that the crystalline undulators discussed in this work can serve as a new efficient source for high energy photons. As we have shown above, the present technology is nearly sufficient to achieve the necessary conditions to constract not only crystalline undulator, but also achieve the stimulated photon emission regime. The parameters of the crystalline undulator and the radiation generated with its use differ substantially from what is possible to achive with the undulators based on magnetic fields.

In our opinion the effects described above is worth experimental study. As a first step, one may concentrate on measurements of the spontaneous undulator radiation spectra.

The related problems not discussed in this paper, but which are under consideration, include the investigation of the crystal damage due to the acoustic wave, photon flux and beam propagations.

\section{References}

1. A.V. Korol, A.V. Solov'yov and W. Greiner, J. Phys. G 24, L45 (1998).

2. A.V. Korol, A.V. Solov'yov and W. Greiner, Int. J. Mod. Phys. E 8, 49 (1999).

3. U.Mikkelsen and E.Uggerhøj, Nucl. Inst. and Meth. B 160, 435 (2000).

4. A.V. Korol, A.V. Solov'yov and W. Greiner, Int.J. Mod.Phys. E 9, 77 (2000).

5. D.S. Gemmel, Rev. Mod. Phys. 46, 129 (1974).

6. V.M. Biruykov, Y.A. Chesnokov and V.I. Kotov, Crystal Channeling and its Application at High-Energy Accelerators (Springer, Berlin, 1996).

7. W.Krause, A.V. Korol, A.V. Solov'yov and W. Greiner, J. Phys. G 26, L87 (2000).

8. A.V. Korol, A.V. Solov'yov and W. Greiner, J. Phys. G 27, 95 (2001).

9. V.N. Baier, V.M. Katkov and V.M. Strakhovenko, High Energy Electromagnetic Processes in Oriented Single Crystals (World Scientific, Singapore, 1998).

10. C.Caso et al, Review of Particle Physics, Eur. Phys. J. C 3, (1998).

11. J. Lindhard, K. Dan., Viddensk. Selsk. Mat. Phys. Medd. 34, 14 (1965)

luederitz: submitted to World Scientific on November 13, 2018 